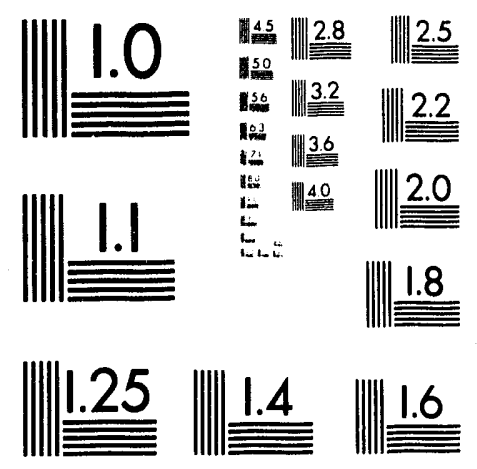



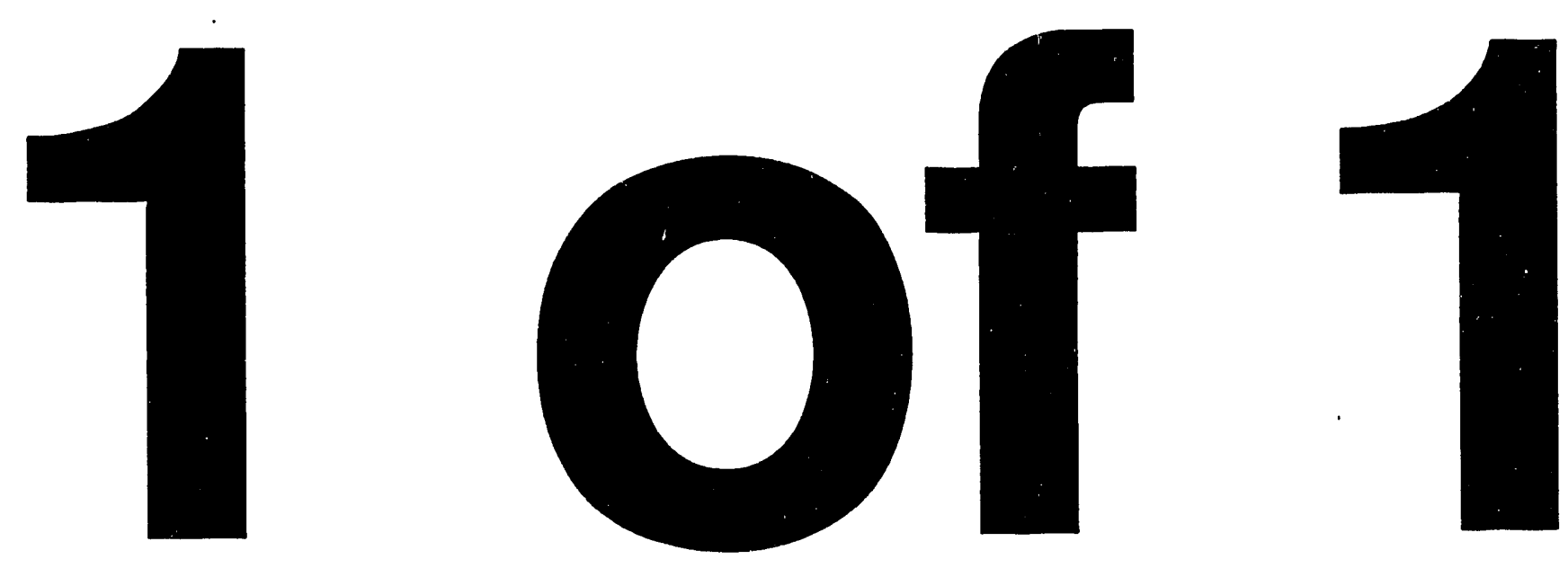


\title{
Conceptual Design of the Neutral Beamline for TPX Long Pulse Opération
}

\author{
K.E. Wright, F. Dahlgren, H.M. Fan, L.R. Grisham, F.G. Hammersand, \\ J.H. Kamperschroer, L.M. Lontai, M.E. Oldaker, P. Rogoff \\ Princeton Plasma Physics Laboratory \\ P. O. Box 451, Princeton, New Jersey 08543
}

\begin{abstract}
The Tokamak Physics Experiment (TPX) will require a minimum of $\mathbf{8 . 0}$ megawatts of Neutral Beam heating power to be injected into the plasma for pulse lengths up to one thousand (1000) seconds to meet the experimental objectives. The Neutral Beam Injection System (NBIS) for initial operation on TPX will consist of one neutral beamline (NBL) with three ion sources. Provisions will be made for a total of three NBLs. The NBIS will provide $5.5 \mathrm{MW}$ of $120 \mathrm{keV} \mathrm{D}^{0}$ and 2.5 $M W$ of partial-energy $D^{0}$ at $60 \mathrm{keV}$ and 40 keV. The system also provides for measuring the neutral beam power, limits excess cold gas from entering the torus, and provides independent power, control, and protection for each individual ion source and accelerating structure. The Neutral Beam/Torus Connecting Duct (NB/TCD) includes a vacuum valve, an electrical insulating break, alignment bellows, vacuum seals, internal energy absorbing protective elements, beam diagnostics and bakeout capability. The NBL support structure will support the NBL, which will weigh approximately 80 tons at the proper elevation and withstand a seismic event. The NBIS currently operational on the Tokamak Fusion Test Reactor (TFTR) at the Princeton Plasma Physics Laboratory (PPPL) is restricted to injection pulse lengths of two (2) seconds by the limited capability of various energy absorbers. This paper describes the modifications and improvements which will be implemented for the TFTR Neutral Beamlines and the NB/TCD to satisfy the TPX requirements.
\end{abstract}

\section{INTRODUCTION}

The function of the Neutral Beam Injection System (NBIS) [1] is to inject hot hydrogen or deuterium atoms into a hydrogen or deuterium plasma. Hydrogen or deuterium ions are generated by passing an electric arc discharge through low-pressure hydrogen or deuterium gas to form a plasma within the source. The ions are extracted and accelerated to a specified energy level by electric fields. The extracted ions are charge-exchanged neutralized on a gas target of sufficient density to obtain a near equilibrium ratio of ions to neutrals. The unneutralized particles are deflected out of the beam by a deflection magnet and directed onto an ion-beam dump. The beams of energetic neutral particles penetrate the confining magnetic fields of the tokamak and interact with the plasma.

The NBIS will provide $5.5 \mathrm{MW}$ of $120 \mathrm{keV} \mathrm{D}$ and $2.5 \mathrm{MW}$ of partial-energy $\mathrm{D}^{\circ}$ at $60 \mathrm{keV}$ and $40 \mathrm{keV}$. The overall NBL and NB/TCD is shown in Figure 1. The system also provides for measuring the neutral beam power, limits excess cold gas from entering the torus, and provides independent power, control, and protection for each individual ion source and accelerating structure.

The duct connecting the NBL to the torus includes a vacuum valve, an electrical insulating break, alignment bellows, vacuum seals, internal energy absorbing protective elements, beam diagnostics and bakeout capability.

The TFTR neutral beamline has been designed by the Lawrence Livermore National Laboratory (LLNL) [2] and the Long Pulse Ion Sources were developed by the Lawrence Berkeley Laboratory (LBL) [3]. LLNL and LBL engaged in a joint project which included the design, fabrication, and testing of a prototype beamline. A "production" beamline was operated in a test stand at PPPL for several years and four (4) NBLs have been operated on TFTR since 1984. These NBLs, which were initially operated with half-second ion sources, were upgraded in 1986-87 for pulse lengths up to two (2) seconds with the Long Pulse Ion Sources (LPIS) [4,5,].

The existing energy absorbing components i.e., beam defining scrapers, ion-beam dump and calorimeter, in the TFTR NBLs are simple water cooled copper plates which were designed to absorb the incident edge beam power by means of their thermal inertia for two seconds with a two and one-half minute cool down interval between pulses. These components must be replaced with an actively cooled design for 1000 second TPX pulses [6]. While several $\mathrm{cw}$ designs were considered, the hypervapotron elements currently being used on the Joint European Torus (JET) beamlines were chosen due to their lower cooling water demands and reliable performance on JET.

Installation of these hypervapotrons will require enlarging several regions within the beamlines, the high elevation of TPX's centerline necessitates a new support structure, the NB/torus connection duct will be reconfigured to include actively cooled protective liners and be compatible with the port(s) on the TPX Tokamak, several additional protective plates will be installed within the beamline, diagnostic capability will be upgraded, the power cables to the LPIS must be water cooled and increased cooling water capacity must be available. 


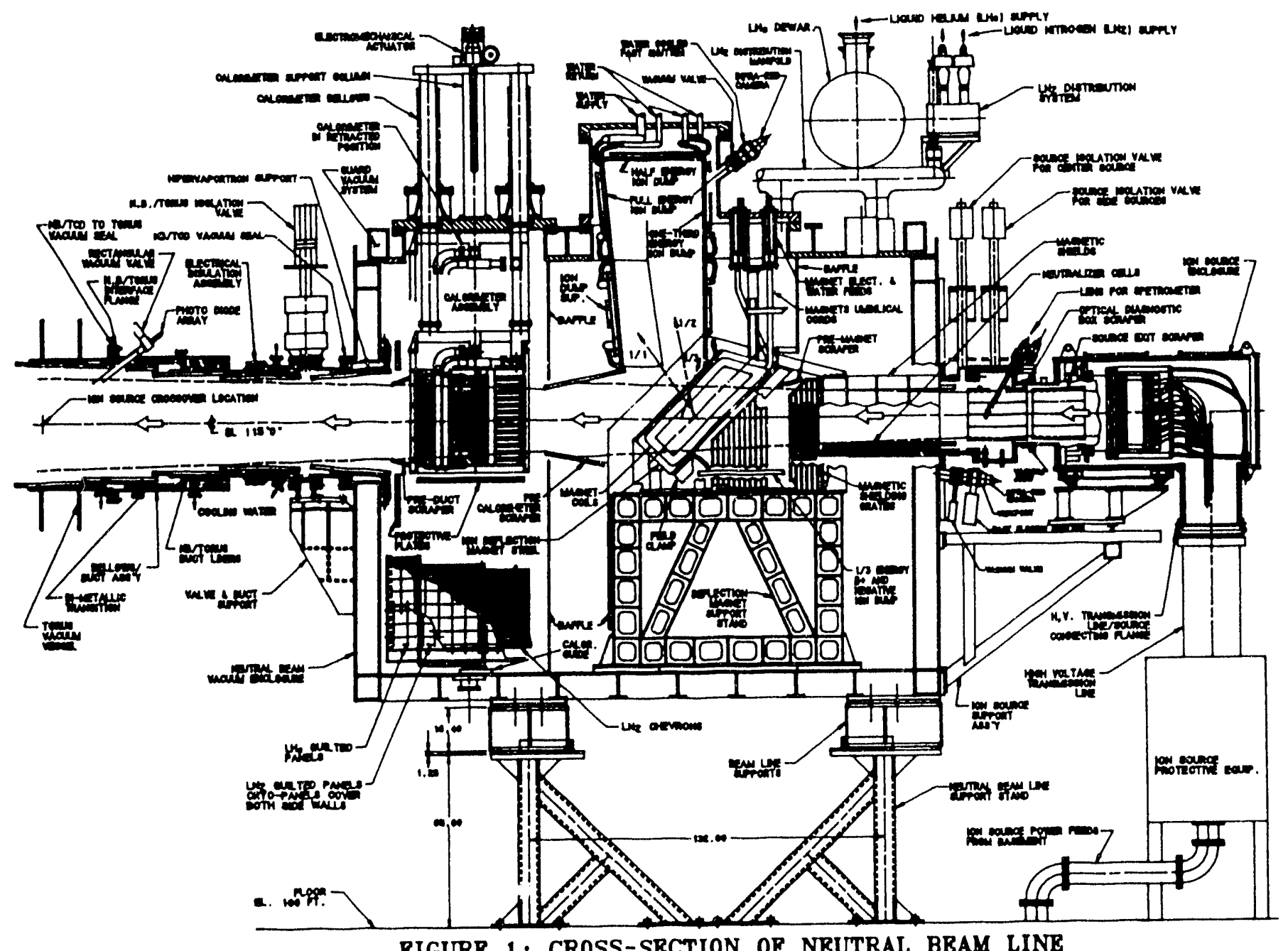

\section{PIGURE 1: CROSS-SBCTION OF NEUTRAL BBAM LINE}

A significant effort has been made to reuse as much of the existing hardware as possible. All new designs and upgrades are based on PPPL's and JET's operational experience and will utilize existing tochnologies.

\section{LONG PULSE ION SOURCE}

The LPIS consists of a Plasma Source in which the injected hydrogen or deuterium gas is ionized, a Magnetic Bucket Assembly which confines the ions via a magnetic field to enhance the plasma uniformity and species mix, a Probe Plate with a set of six Langmuir probes to monitor plasma parameters, and an Accelerator Assembly which focuses and accelerates the ions.

The LPIS is mounted inside an enclosure on a gimbaled aiming mechanism which can rotate the axis of the emitted beam by $\pm 0.5^{\circ}$ in both the horizontal and vertical planes for proper transmission through the NB/TCD. The ion source enclosure is made of low-carbon steel and contains a liner of moly-permalloy, which is used for shielding the LPIS from the tokamak magnetic ficlds. The interior of the enclosure is filled with 12-15 psig gaseous sulfur hexafluoride to improve the electrical holdoff capability. See Figure 2.

A Source Exit Scraper, located inside the bellows assembly within the aiming mechanism, will intercept an excessively divergent extracted beam from the Accelerator and will confine the beam transmitted through the scraper to one having a $12.78 \mathrm{~cm}$ horizontal by $42.22 \mathrm{~cm}$ vertical cross-section. The vacuum flange on the exit end plate of the Source Enclosure will be enlarged to permit installation of the cw Scraper Assembly and an electrical feed-thru will be added. Two electromagnetic bucking coils, each with 20 Amp-turns separately energized, will be installed around the cuffs at each end of the bellows to cancel residual axial magnetic ficlds up to 500 milligauss established in the output of the LPIS by imperfectly shielded stray fields.

The Electron Dump in the Plasma Source provides a back end closure for the magnetic multicusp plasma chamber and intercepts primary electrons from the filaments, radiation from the plasma, and backstreaming electrons from the accelerator gaps. For the two second pulse lengths required on TFTR, this dissipation was accomplished primarily through the use of the thermal inertia in a thick copper dump. However, for longer pulse lengths on TPX, active water cooling will be necessary [7]. The Langmuir Probes currently in use on TFTR were designed for $\mathrm{cw}$ operation. Despite extremely small spacing in the water courses, the probes have operated reliably. Failures, when they occurred, have either been due to extremely small leaks which allow water vapor to penetrate into the LPIS vacuum environment or electrical shorts across the probe tip due to an accumulation of tungsten on the insulator. "Button Type" Probes, which 
will not expose any insulator surface to the plasma and be less prone to damage, will be used in TPX [7].

For TPX, the filaments and plasma arc will be energized by conducting wic current through a system of water-cooled cylindrical buses that enter the source Enclosure through a cylindrical port [8]. The positive and negative water cooled buses will be individually terminated in connector plates located above the bottom of the Housing Assembly as shown in Figure 2. Individual flexible cables will be used to carry the current from the water cooled buses to pin contacts on the source. The number of cables has been increased to provide an overrating for operation on TPX. The existing cables and connectors will be used on TPX by cutting them shorter and brazing them tc he new bus connector plates.

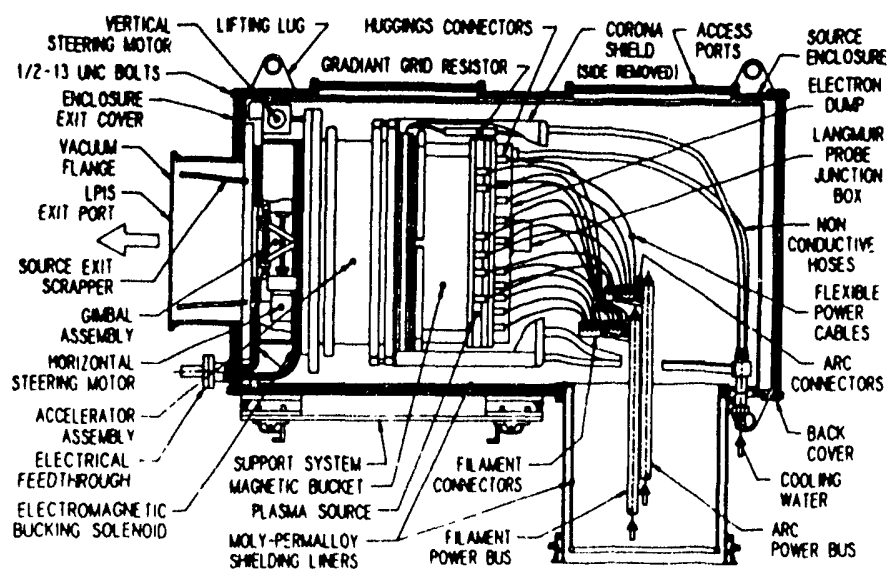

LONG PULSE ION SOURCE INSIDE ENCLOSURE

The TPX NB Gas Injection System (GIS) must supply Hydrogen $\left(\mathrm{H}_{2}\right)$ or Deuterium $\left(\mathrm{D}_{2}\right)$ gas to three LPISs, for conditioning, injection, and neutralization. To ensure proper source operation and maintain optimum line density in the neutralizer region, a precise regulation of throughput must be maintained. Independent GIS subsystems will be installed on each LPIS and Optical Multichannel Analyzer (OMA) chamber within the neutralization region to provide operational flexibility and permit the minimization of gas throughput, thereby maximizing the interval between regenerations of the cryogenics panels. Each subsystem must be capable of delivering a throughput of 50 torr-1/sec of $\mathrm{H}_{2}$, or 40 torr- $1 / \mathrm{sec}$ of $\mathrm{D}_{2}$ for the full duration of the pulse. A closed loop controller will be used to regulate the flow rate by controlling the voltage to the piezoelectric valve as a function of plenum pressure. The LPIS and OMA GIS will be at ground potential. The OMA gas feedpoint would be used for the possible option of injecting tritium gas, using the existing $\mathrm{T}_{2}$ system design. Relatively minor reconfiguration of the existing TFTR NB gas system [9] will permit its use on TPX.

\section{NEUTRAL BEAM MODIFICATIONS}

The NB vacuum enclosure, the cryocondensation vacuum pumping system and the three-gap ion deflector magnet along with its support stand will be used as is. However, several modifications are required to permit installation of the actively cooled energy absorbers and upgrade the diagnostic capability.
Each of the three ion sources on the NBL must be isolated from the NB vacu'im enclosure to permit replacement of a complete ion source assembly without letting the rest of the system up to air. The Source Isolation Valves (SIV) utilized on the TFTR Neutral Beamline have a clear aperture of $500 \mathrm{~mm}$ diameter. This opening must be enlarged to allow installation of the hypervapotron scrapers required for $1000 \mathrm{sec}$ long pulse operation on TPX. These new SIVs will be $630 \mathrm{~mm}$ diameter single gate type manufactured by VAT, Inc. with a single viton, Type E-60C elastomer o-ring as the gate seal. All other seals used in the valve will be metallic. Since these $630 \mathrm{~mm}$ valves will not physically fit side by side within the space provided by the three sources being aimed at an angle of $4.039^{\circ}$ between them, the valves will be staggered along the beam trajectory; i.e., the two outside valves will be located further away from the $90^{\prime \prime}$ flange on the neutral beam enclosure. This nesting, along with additional space required for the larger source housing vacuum seal, requires the center ion source to be moved further away from the cross-over location by $6.75^{\prime \prime}$ and the two outside sources to be moved 12.0" further away from the cross-over location. See figure 3.

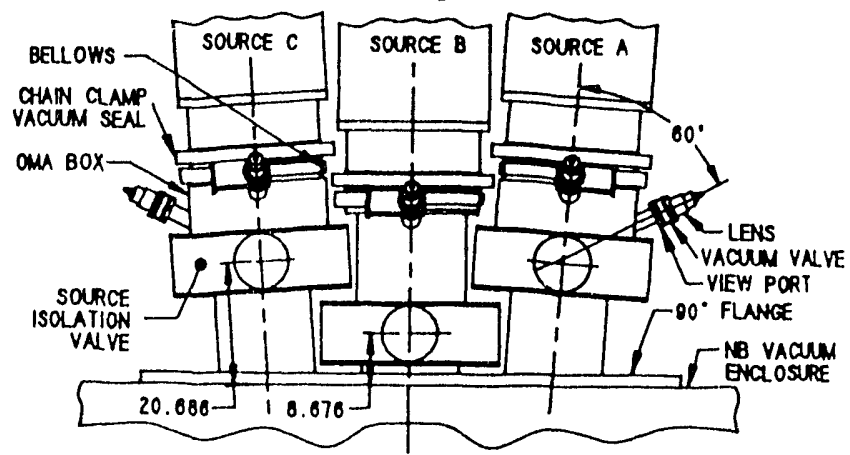

PIGURB 3 SOURCB ISOLATION VALVBS IN STAGGBRRD POSITION

A chamber, or box, is located at the exit of each of the three ion source enclosures to obtain diagnostic information of the extracted beam by Optical Multichannel Analyzers (OMA). The chambers will be a rectangular parallel-piped vacuum enclosure capable of holding a vacuum of $\leq 1 \times 10^{-8}$ torr. Different size chambers will be used on the central and side beams to compensate for the required SIV offsetting. The bottoms, sides and top of the boxes will be fabricated from mild steel to provide magnetic shielding of the beam while the ends will be nonmagnetic stainless steel to avoid drawing magnetic flux inwards toward the beams. A liner of moly-permalloy will be installed inside the enclosure to further reduce the magnetic field through which the beam must pass. The entrance port to the OMA will contain a bellows to permit alignment during ion source installation and a vacuum flange which makes up one-half (the other half is on the source enclosure) of the OMA/source enclosure seal. The flanges are clamped together by a quick-release chain clamp to form a vacuum-tight connection.

Viewing ports angled at $60^{\circ}$ to the beam centerline will be included in the lids of all three OMAs and on the sides of the two outside OMAs. A $63 \mathrm{~mm}$ diameter vacuum valve, a quartz viewpoint and a spectrometer pickup will be mounted on each port. The viewing 
- window is external to the valve so that the valve can be closed to minimize coating the quartz from sputtering and permit cleaning and/or replacement without letting the rest of the system up to air. These ports are angled such as to accept light radiated from the beams along a path lying in the vertical or horizontal plane, but inclined such that the frequency is Doppler shifted down in wave length. Light is sent through fiber optics to a spectrometer. The angles are chosen so that three peaks are observed, one for each energy $(120,60$, and $40 \mathrm{keV})$. Relative intensities provide an indication of species mix; broadening of the peaks indicates the source angular divergence.

The ions extracted from the sources are chargeexchange neutralized on a gas target of sufficient density to obtain a near equilibrium ration of ions to neutrals. The neutralizing target must be the same gas as the LPIS is emitting. The neutralizer region extends from the LPIS emitting grid to the end of a rectangular duct $120 \mathrm{~cm}$ beyond the $90^{\circ}$ flange and has an intemal aperture 15.32 $\mathrm{cm}$ wide by $50.32 \mathrm{~cm}$ high, which is the same aperture as the neutralizer cells utilized on TFTR. The internal surfaces of the neutralizer cells will be hypervapotron elements which are capable of adsorbing divergent beam power. These hypervapotrons will be surrounded by $1 / 8^{\prime \prime}$ moly-permalloy, a $1 / 4^{\text {n }}$ thick soft-iron rectangular tube and $1 / 2^{n}$ thick soft-iron picture-frame sections to provide magnetic shielding for the traversing ions. This magnetic shielding will be of the same design as utilized on TFTR except it must be of larger cross-section to fit around the hypervapotrons.

The lid over the ion dump will be converted to a "high-hat" configuration to provide more height for the extended full energy ion dump. Three viewing ports will be installed in the cylindrical portion to permit viewing the dump with infrared cameras. Three ports will also be installed in the $90^{\circ}$ flange to similarly view the calorimeter.

- The calorimeter is raised by an electromechnical actuator to allow beam firing into the torus. All three vees

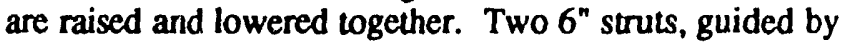
bushings and sealed with a $38^{\prime \prime}$ stroke bellows, contain the water inlet and outlet pipes. When the calorimeter is to be removed from the vessel, it is guided upward by rollers riding on vertical posts permanently installed within the beamline so it will pass through the $52^{\prime \prime}$ hatchway. During operations on TFTR, problems occurred due to the bellows being located inside the NB vacuum enclosure. Therefore, the external surfaces were exposed to cryogenic surfaces while the internal surfaces were exposed to atmospheric conditions. This configuration enhanced "squirming" of the bellows due to pressure loading and resulted in condensation and freezing of moisture from the atmosphere inside the bellows. The ice caused cracks to develop in the outside diameter welds between convolutions when the bellows were compressed. These conditions will be alleviated on the TPX NBL by installing the bellows external to the NB vacuum enclosure. This redesign will also locate the actuator in a position where it will lift the vees in lieu of pushing them up as presently configured. This action will permit the vees to be guided better during travel and avoid binding due to misalignment.
The water outlet temperature of each hypervapotron panel in the ion dump and calorimeter is measured by thermocouple probes inserted into thermocouple wells which are welded into the outlet lines. Water calorimetry can be performed on each individual hypervapotron. The probes and wells are commercial items while the adapter fittings for the wells are custom designed for the application. The hypervapotron front plate temperature, approximately $0.125^{\prime \prime}$ from the heated surface will be measured directly by sub-miniature commercial thermocouple probes. The probes will be inserted into holes drilled down through the panel sidewall into the comer area of the front face.

Based on inspections of internal components within the TFIR NBLs, several protective plates which do not currently exist will be required for 1000 second duration beam pulses on TPX. The locations of these protective plates include the top and bottom of the magnet yoke, the magnet's pole faces and coils, below the magnet entrance, before the pre-calorimeter scraper, above and below the calorimeter "vees" and in front of the exit end of the NB enclosure. While the total power deposited at these locations is not a large percentage of the extracted ion beam, magnetic focusing can result in very high localized heat loads. Therefore, hypervapotron elements rated for $\mathrm{cw}$ operation will be used for these protective plates in lieu of thermal inerial protectors.

\section{NB/TORUS CONNECTING DUCT}

The primary function of the NB/TCD is to provide a vacuum boundary of ample size through which the beams of energetic neutral particles frc : all three ion sources in the neutral beam can pass into the wrus without deleterious effects from the beams striking the duct walls. The NB/TCD must also include components to isolate the neutral beam vacuum enclosure from the torus vacuum vessel, electrically insulate the NBL from the torus, permit alignment of the NBL, and allow for the excursions of the torus interface during both bakeout up to $350^{\circ} \mathrm{C}$ and under operational conditions.

A high vacuum valve with a clear opening of one $m^{\wedge}$ 'er diameter is required between the torus and the NB vacuum enclosure to provide a seal which will have a maximum total leakage of $10^{-9}$ torr-1/sec of helium, when either vessel is under vacuum while the other is at atmospheric pressure. The valve is a single gate type manufactured by VAT, Inc. with a single Viton, type E$60 \mathrm{C}$ elastomer o-ring as the gate seal. All other seals used in the valve are metallic. The valve is bakeable in either the open or closed position to $150^{\circ} \mathrm{C}$ inside temperature without exceeding the specified leak rates. Four of these valves have operated without failure during the entire TFTR operations. Since the design lifetime is 20,000 cycles between servicing, trouble-free operation on TPX can be expected.

The electrical insulating break must have a minimum inside diameter of $90 \mathrm{~cm}$, be bakeable at $150^{\circ} \mathrm{C}$, withstand atmospheric pressure, and standoff $8.6 \mathrm{kV}$. The electrical insulator assemblies used successfully on TFTR are composed of three circular alumina ceramic rings, brazed to AISI Type 430 stainless steel spinnings. The spinnings, 
which have a cross-section shaped like a "J", of these brazed assemblies are then welded to transition rings which are subsequently welded in place to the connecting duct flanges on each side of the insulator. A second larger diameter porcelain ring is used to create an intermediate vacuum region for leak checking and monitoring. For installation in the TPX NB/TCD, the duct transition section on the torus side of the TFTR insulator assembly will be removed by machining away the weld joint between the transition ring and the transition duct flange and subsequently, unbolting the insulator assembly flanges. A new transition duct which satisfies TPX spatial and operating requirements will then be installed.

A bellows assembly is required to allow alignment of the NBL during installation onto the torus and torus deformations due to bakeout, design pressure, plasma disruption; and seismic load. It must also be capable of supporting atmospheric pressure, have a maximum total leakage of $10^{-9}$ torr- $1 / \mathrm{sec}$ of helium, and be bakeable to $250^{\circ} \mathrm{C}$. The bellows assembly must withstand an axial compression of $1.6 \mathrm{~cm}$, a vertical displacement $\mathrm{cit} 1.0 \mathrm{~cm}$, and a lateral displacement of $0.7 \mathrm{~cm}$ due to thermal expansions when the torus is baked to $35 C^{\circ} \mathrm{C}$. There will also be smaller deflections during each tokamak pulse. Therefore, the bellows must be designed for adequate fatigue life for bakeout cycles, pressure cycles and a high number of tokamak pulse cycles. It must have a rectangular cross-section with a minimum inside clearance of $106.7 \mathrm{~cm}$ vertically and $76.2 \mathrm{~cm}$ horizontally. Preliminary analysis using a finite element model indicates that all requirements can be achieved with a bellows assembly consisting of two identical welded bellows, each $20.32 \mathrm{~cm}\left(8^{\prime \prime}\right)$ long with sixteen $0.078 \mathrm{~cm}\left(0.031^{\prime \prime}\right)$ thick, $6.1 \mathrm{~cm}\left(2.4^{\prime \prime}\right)$ high convolutions, separated by a $30.5 \mathrm{~cm}$ (12") long spacer section. The height of the convolutions is restricted by the TF coils and cryostat assembly. Even though the TPX bellows is larger (10" higher and 6.5" wider) in cross-section than the TFTR NB/TCD bellows, this analysis indicates that the lateral stiffness will be much less since the overall length is substantially larger. In addition, the bellows-duct assembly is supported by four rods which support the dead weight and improve the stability of the assembly. The bellows and duct spacer assembly will be fabricated of type 625 Inconel to utilize proven technology.

The NB Duct/Torus Interface Flange must provide a compatible sealing surface for making up a removable bolted rectangular vacuum tight seal with a leak rate $\leq 10^{-9}$ torr- $1 / \mathrm{sec}$ of helium plus provide a transition to the NB/TCD Bellows Assembly. This flange and transition section will be fabricated of titanium to avoid differential thermal expansions during bakeout up to $350^{\circ} \mathrm{C}$ and under TPX operational conditions. The transition section will contain an explosively welded piece that bonds titanium to Nitronic 33 to which the Inconel 625 bellows can be welded.

The vacuum seal between the $N B / T C D$ flange and the torus interface flange will be a double metallic seal with provisions to evacuate the interstitial volume between the two seals to facilitate leak-checking and provide for a "guard vacuum" system. The effective leak rate from the atmosphere to the internal vacuum region can be reduced several orders of magnitude by evacuating the region between two seals. This technique is employed throughout the NB/TCD and the Neutral Beam Vacuum Enclosure. The vacuum seal utilized elsewhere in the NB/TCD will have a similar internal metallic seal, but the external seal will be Viton E-60C. The use of an elastomer for the external "guard vacuum" seal reduces the total seal line load and permits locating the bolts external to both seals, thereby eliminating the necessity for seals under the bolt heads. The selection of these seal designs for TPX is based on several years of trouble free service on TFTR as well as use in the reactor vessel of nuclear power plants.

During bakeout, the temperature at the torus port is $350^{\circ} \mathrm{C}$ while the temperature of the electrical break and vacuur, valve must be kept at $150^{\circ} \mathrm{C}$. Some cooling will be provided to cool sections of the Inconel transition duct between the explosively bonded joint and the first bellows from $350^{\circ} \mathrm{C}$ to $250^{\circ} \mathrm{C}$, and to cool the transition duct between bellows from $250^{\circ} \mathrm{C}$ to $150^{\circ} \mathrm{C}$. The bellows will be bakeable by a heating system that is thermally insulated to minimize heat loss and help equalize the bellows temperature along its length. This heating jacket must be easily removable so that the duct connecting this bellow assembly to the torus can be unbolted.

Based on analysis of TFTR neutral beam transport [6], it is estimated that roughly $4 \%$ of the total neutral power is lost in the NB/TCD. This amount of power would not present a significant heat load deposition on the duct walls if it is uniformly distributed. Deposition of this power in the TPX NB/TCD must be avoided for the required long pulse operation. The pre-calorimeter and pre-duct scrapers must be sized to provide complete shadowing of all duct surfaces. "Scraping off" the most divergent neutral beam must be accomplished within the neutral beam vacuum enclosure to take advantage of the cryo-condensation pumping panels and minimize the pressure within the NB/TCD.

Beam-blocking could possibly result from reionization of the neutral beam due to outgassing from the inner surfaces of the NB/TCD. The restrictive aperture of the NB/TCD, which is only $800 \mathrm{~mm}$ vertically by 400 mm horizontally, must transmit $>8 \mathrm{MW}$ of neutral power. A NB/TCD duct with well conditioned copper liners can be expected to operate well below the beam-blocking threshold at full power. However, the quantity and location of deposition of re-ionized particles is unpredictable for varying operational scenarios. Fast response Penning gauges will be installed in the NB/TCD to directly measure the pressure within the duct and provide an interlock signal. The top of the transition section will contain a rectangular port at $45^{\circ}$ to the beam's horizontal axis and a flange for mounting a rectangular vacuum valve with a $25 \mathrm{~mm}$ by $420 \mathrm{~mm}$ aperture and a view port. This rectangular opening shall provide a line of sight across the beam crossover location. Optical diagnostics will be employed to ascertain proper aiming of the ion sources since any misalignment of the beams could result in serious damage in this restrictive region. Based on experience at JET, plus the above considerations, the entire internal surface of the NB/TCD will be covered with hypervapotron heat absorbing panels. These duct liners will consist of five subassemblies of varying cross-sections distributed axially 
along the trajectory. All hypervapotrons within the $\mathrm{NB} / \mathrm{TCD}$ shall have both the water inlet and discharge at the upstream beam end to allow for protection or shadowing of all plumbing. Transitions between the sections will be accomplished by overlapping ends, thereby eliminating the possibility of any beam impingement normal to the leading edges. The sides of all hypervapotrons within the NB/TCD will be contoured to prevent a direct line-of-sight for particle transmission.

\section{NEUTRAL BEAM SUPPORT STRUCTURE}

The TPX geometry requires that the NBL, which weighs about 80 tons, will have to be raised about 65 inches from the present TFTR floor level in order for the system to align with the mid-plane of the torus at operating temperature.

The primary design criteria is the seismic environment at the Test Cell elevation of the most intense earthquake (MIE) conditions as specified in the TPX General Requirements Document. The maximum accelerations are between 5 to $8 \mathrm{~Hz}$ for the principal axes of the excitation. The NBL structure (calculated for the existing TFTR configuration) exhibits primary natural frequencies in the range of 19 to $21 \mathrm{~Hz}$. Therefore, it is reasonable to design the NBL-TPX support structure for primary frequencies above $\approx 24 \mathrm{~Hz}$ to eliminate any possible dynamic excitation amplification. This stiff support structure would also limit the relative motion between the NBL and the Torus Vacuum Vessel, which will aid in the design of the connecting duct and bellows.

A finite element model was created to study and size the necessary support structure. In this simplified model, the support structure was simulated via bar elements while the Neutral Beam Enclosure was considered rigid. The results show the first (lateral) mode shape at $22.54 \mathrm{~Hz}$ and the second (longitudinal) mode shape at $26.28 \mathrm{~Hz}$. The third mode shape is rotational at $85.87 \mathrm{~Hz}$ and is not important for the design of this structure since the seismic loads at this frequency are negligible. This model will be changed to a 3-dimensional (plate elements) formulation for the final optimized design process.

The NBL support structure will consist of four $8^{\text {" }}$ by $12^{n}$ rectangular columns, eight $8^{n}$ by $8^{n}$ corner braces, and eight $8^{\prime \prime}$ by $8^{\prime \prime}$ square truss braces fabricated from one inch thick plate that is welded to form four rigid corner trusses. Floor plates will be used to secure the entire structure to the Test Cell floor.

The estimated total weight of the support structure is $\approx 12,500$ pounds. Columns, braces, floor plates, and gussets will be assembled from $1.0^{\prime \prime}$, and, the leg platforms are $2.0^{\text {" }}$ thick plates. The support structure will be made of 304 stainless steel and requires about 7,840 inches of full penetration welds.

\section{SUMMARY}

The TFTR NBL(s) will be upgraded for operation on TPX by replacing the existing energy absorbing components with actively cooled hypervapotron elements. Various components along the beam trajectory will be enlarged to provide additional space required for this installation. Only a few relatively minor upgrades are necessary for the ion sources to operate $\mathrm{cw}$. The duct connecting the NB to the torus will reuse an existing vacuum valve, spool sections and electrical insulating breaks. However, a new bellows will be required to allow for larger excursions of the TPX beam eatrance duct. A new transition section will interface with the torus flange and avoid differential thermal expansions at vacuum seals. The NB/TCD will be lined with hypervapotron elements to enhance beam transmission. The NB diagnostics will be upgraded to ensure proper beam alignment and provide the capability to perform an energy inventory. All new designs and upgrades are hased on operational experience and existing technologies, thereby minimizing the R\&D necessary, engineering required and initial operating difficulties.

\section{ACKNOWLEDGMENT}

The authors wish to acknowledge the design efforts of Jeff Barnard, Paul Kan, Charles Bromley, Charles Peters and Egidio Mazzuca of the PPPL CAD Division.

This work was supported by US DOE Contract No. DE-AC02-76-CHO3073.

\section{REFERENCES}

[1] A. von Halle et al., "TPX Neutral Beam Injection System Design," this conference.

[2] L.C Pittenger et al., "A Neutral Beam Injection System of the Tokamak Fusion Test Reactor," in Proceedings of the 7th Symposium on Engineering Problems of Fusion Research, 1977, Vol. 1, pp. 555559.

[3] M.C. Vella et al., "Development and Testing of the U.S. Common Long Pulse Source at $120 \mathrm{kV}$," Review of Scientific Instruments, vol. 59, 2357 (1988).

[4] G.M. Gammel et al., "Performance of U.S. Common Long Pulse Ion Source on the Tokamak Fusion Test Reactor," in Proceedings of the 12th Symposium on Fusion Engineering, 1987, Vol. 2, pp. 1137-1140.

[5] T. Stevenson et al., "Operation of the TFTR Neutral Beam Injection System which Achieved Greater than 30 Megawatt Injection," in Proceedings of the 13th Symposium on Fusion Engineering, 1989, Vol. 1, pp. 292-295.

[6] F. Dahlgren et al., "TPX/TFTR Neutral Beam Energy Absorbers," this conference.

[7] R. P. Wells T.A. Stevens, "Modifications of the Long Pulse Ion Source for Operation on the Tokamak Physics Experiment (TPX)," this conference.

[8] S. Ramakrishnan et al., "Neutral Beam Power System for TPX," this conference.

[9] K.E. Wright et al., "Pre-Operational Test Results of the TFTR Neutral Beam D-T Gas Delivery System," in Proceedings of the Tenth Topical Meeting on the Technology of Fusion Engineering, 1992. 

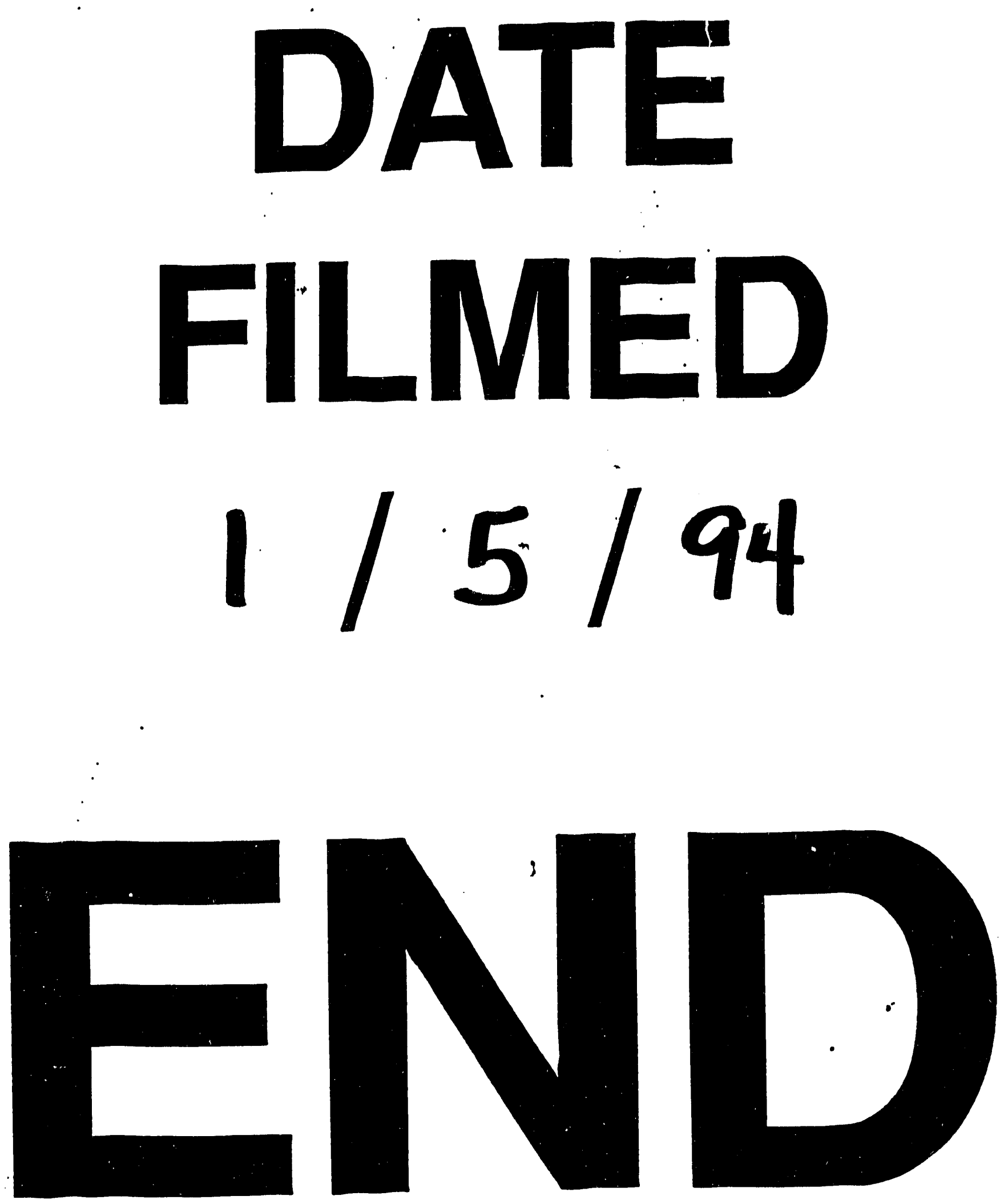
